\title{
Experimental study on flow characteristics of tetrahydrofuran hydrate slurry in pipelines
}

\author{
Wuchang Wang ${ }^{1 *}, \quad$ Shuanshi Fan ${ }^{2}, \quad$ Deqing Liang ${ }^{3}$, Yuxing $\mathrm{Li}^{1}$ \\ 1. Department of Storage and Transportation Engineering, China University of Petroleum, Qingdao 266555, Shandong, China; \\ 2. South China University of Technology, Key Laboratory of Enhanced Heat Transfer and Energy Conservation, MOE, Guangzhou 510640, Guangdong, China; \\ 3. Guangzhou Institute of Energy Conversion, Chinese Academy of Sciences, Guangzhou 510640, Guangdong, China;
}

[ Manuscript received October 19, 2009; revised January 26, 2010]

\begin{abstract}
Tetrahydrofuran (THF) was selected as the substitute to study the flow behaviors and the mechanism of the hydrates blockage in pipelines. The slurrylike hydrates and slushlike hydrates are observed with the formation of hydrates in pipeline. There is a critical hydrate volume concentration of $50.6 \%$ for THF slurries and pipeline will be free of hydrate blockage while the hydrate volume concentration is lower than the critical volume concentration; otherwise, pipeline will be easy to be blocked. Fully turbulent flow occurs and friction factors tend to be constant when the velocity reaches $1.5 \mathrm{~m} / \mathrm{s}$. And then, constant values of friction factors that depend on the volume concentrations in the slurry were regressed to estimate the pressure drops of THF hydrate slurry at large mean velocity. Finally, a safe region, defined according to the critical hydrate volume concentration, was proposed for THF hydrate slurry, which may provide some insight for further studying the natural gas hydrate slurries and judge whether the pipeline can be run safely or not.
\end{abstract}

Key words

hydrate slurry; tetrahydrofuran; flow character; flow assurance

\section{Introduction}

Hydrates are ice-like crystalline compounds and are likely to form in offshore condensate pipeline running at high pressure and low temperature. Pressure drops in pipeline increase and even deadly accidents occur for blockage of the pipeline or destruction of the downstream equipments when hydrates form in pipeline. This has resulted in far reaching effects on the petroleum industry since the first discovery of hydrate in natural gas pipeline in 1930s [1].

In order to keep the pipeline in safe region many kinds of methods, such as dewatering, keeping temperature at a safe level and the addition of thermodynamic inhibitors, have been developed throughout the world [2]. However, since 1970s as oil companies have been producing in ever-increasingly unusual environments, such as the north slope of Alaska, Siberia, and more deepwater fields were discovered or brought in production, the limitation of traditional methods in preventing the formation of hydrates is ever-increasingly obvious [3]. So, new methods for hydrate prevention have been developed and the main strategies dealing with hydrate are changing from avoiding the formation of hydrate [1], developing new kinds of economical low dosage hydrate inhibitors (LDHI) and preventing the formation of hydrate plugs in pipelines [4]. The objectives were and still are to better understand the mechanism of formation of hydrate slurry and to characterize its flow properties as well as to develop methods preventing the formation of hydrate plugs [1].

Understanding the flow characters of hydrates slurry in pipeline is very necessary to prevent the formation of plugs and run pipeline systems safely [5]. For several years, the problem of hydrates in petroleum production has been studied worldwide in laboratory and loop test facilities. Hydrate structures in pipeline were classified into five classes, including slurrylike hydrates, slushlike hydrates, powderlike hydrates and two transitional structures [6]. Rheological behaviors of hydrate in gas/water/Conroe crude system [7] and in dodecane emulsion [8] were studied. In the cases of the formation of the hydrate blocks and the plugs, agglomerations of hydrate particles are thought of playing a key role [8].

However, there are short of systematic researches on morphology and flow behavior of hydrate slurry with different solid volume contents because of the complicated components of oil. This information is very important to ensure the

\footnotetext{
* Corresponding author. Tel: 15863015036; E-mail: wangwuchangupc@126.com
} 
pipeline from blocking [9]. While on the other hand, tetrahydrofuran (THF), which is a kind of solvent and can form hydrate of structure II at atmospheric pressure, has been proved to be a good material in exploratory works of hydrate slurry [10]. So in this paper, THF was chosen as the substitute to study the flow assurance of hydrate slurry on the newly built loop. In the rest of this article, the morphologies as well as the flow characteristics of THF hydrate slurries are presented in detail.

\section{Experimental}

Experiments on flow characteristics of hydrate slurry were performed on the flow loop in Guangzhou Institute of Energy Conversion (GIEC, China). This flow loop (Figure 1), which is a two-pass loop consisting of a $42.0 \mathrm{~mm}$ diameter pipe, $30.0 \mathrm{~m}$ long, was specially built to perform experiments on hydrate slurry at low pressure (no more than 1.5 MPa). The flow loop is enclosed in a temperature chamber $(4.83 \times 3.30 \times 2.55 \mathrm{~m})$, which can keep a stable environment with a temperature between $-40{ }^{\circ} \mathrm{C}$ and $80^{\circ} \mathrm{C}$. More details about the flow loop can be found elsewhere [11].

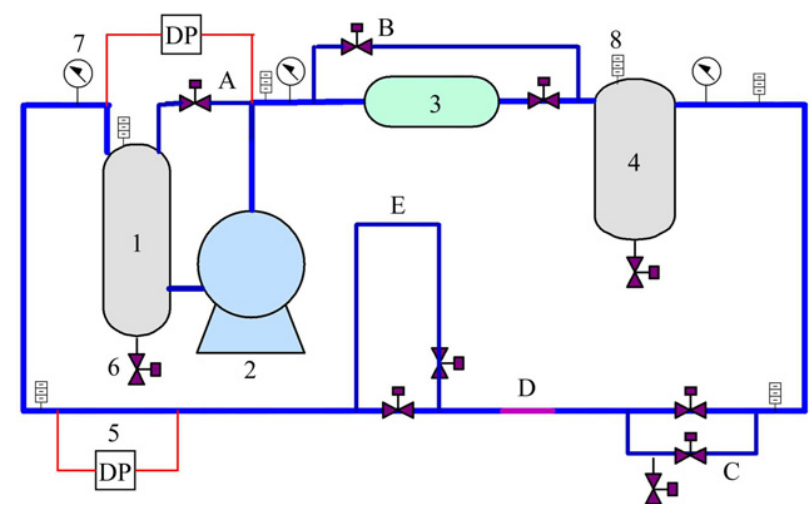

Figure 1. Scheme of the flow loop. 1-Tank, 2-Magnetic centrifugal pump, 3-Flowmeter, 4-Buffer tank, 5-Difference pressure sensor, 6Drain valve, 7-Pressure sensor, 8-Temperature sensor; A- Sideline, BSideline for flowmeter, C-Dip part, D-View port, E-Vertical part

At the beginning of each experiment, commercial tetrahydrofuran (THF) with a certified purity of $99.994 \mathrm{wt} \%$ and tap water were injected into the material tank according to the assigned volume concentration of THF hydrates. For a $19 \mathrm{wt} \%$ stoichiometric THF-water solution, the equilibrium temperature to form hydrate is $+4.4{ }^{\circ} \mathrm{C}[12-14]$. The equilibrium temperature of hydrate decreases with the decreasing of THF in solution [8]. The pump was started when the temperature in the environmentally controlled room reached the designed value, usually at -2 to $-1{ }^{\circ} \mathrm{C}$, which cooled the fluids in the loop to the equilibrium temperature according to these experiments, and ran at a steady speed until all THF in pipeline was converted into hydrates. The processes of the hydrate formation were observed through the view section and by the samplings of the pipelines. The morphologies did not change for more than one hour and the temperature of the liquid in pipeline began to decrease because of the temperature difference between the liquids and the environment in the chamber when all THF in pipeline was converted into hydrates, which indicated the end of the hydrates formation in pipeline. And then a series of flow experiments of the hydrate slurries were performed. Hydrate volume concentration $\left(\Phi_{\mathrm{h}}\right)$ and the mean velocity $(w)$ were varied from $5 \%$ to $70 \%$ and from 0.5 to $3.6 \mathrm{~m} / \mathrm{s}$ respectively in the experiments.

\section{Results and discussion}

Formation of hydrates changes the morphology, the properties of the fluids in pipeline and volume concentration $\left(\Phi_{\mathrm{h}}\right)$ of hydrates in pipeline. The latter decides the flow characteristics of the slurry. In this paper, a simple but traditional method was used to decide the $\Phi_{\mathrm{h}}$ of the hydrate in pipeline, in which samplings were drawn from the pipeline draining valve and the samples were dissociated to weigh the masses of water $\left(m_{\mathrm{w}}\right)$ and THF $\left(m_{\mathrm{T}}\right)$ in the samples. Now, the volume concentration $\left(\Phi_{\mathrm{h}}\right)$ of the hydrates in pipeline can be calculated as the ratio between the hydrates volume and the sum of hydrates and the residual water [12]:

$$
\Phi_{\mathrm{h}}=\frac{m_{\mathrm{h}} \rho_{\mathrm{w}}}{m_{\mathrm{h}} \rho_{\mathrm{w}}+\left(m_{\mathrm{w}}-m_{\mathrm{wh}}\right) \rho_{\mathrm{h}}}
$$

Where $\rho_{\mathrm{w}}$ is the density of water, $\rho_{\mathrm{h}}$ is the density of hydrate, $m_{\mathrm{h}}$ is the mass of hydrate in the sample, and $m_{\mathrm{wh}}$ is the mass of water consumed in forming hydrates in he sample, both of which can be calculated by $m_{\mathrm{T}}$ according to the hydration number of THF hydrate. The relation of (1) can be used to calculate the volume concentration of the hydrate in pipeline assuming that all the THF in pipeline is consumed in forming hydrate and there are only water and hydrates in pipeline, which is ensured by waiting for a relatively long time and repetitious measurement and observation. Study on the flow properties of the slurry in pipeline was conducted when the $\Phi_{\mathrm{h}}$ in pipeline did not change any more.

\subsection{Morphology of hydrate slurry}

An example of hydrate macroscopic structure in the pipeline is presented in Figure 2. The water cut of mass ratio in the example is $87 \%$ and volume concentration of hydrates is $65 \%$ when all the THF has been converted into hydrates, of which the equilibrium temperature of the THF hydrates is about $4.0^{\circ} \mathrm{C}$ [8]. The six pictures in Figure 2 show the change of macroscopic structures in pipeline as a function of time. The time noted under the picture is logged from the time when the temperature of the mixture decreased below $4.0^{\circ} \mathrm{C}$. The particles of THF hydrate were first observed at about $2 \mathrm{~h}$. Although the hydrates are lighter than the mixed solution, the mixture of hydrate and liquid are homogeneous in pipeline. At about $13 \mathrm{~h}$, the transition from slurrylike hydrate to slushlike hydrate began. And then, more hydrates formed and the solid fraction in the fluid became larger, which resulted in losing the character of the fluid. The velocity of the slushlike hy- 
drates became ever-increasingly slow while keeping the pump at a same motor speed. At last the slushlike hydrates stopped flowing and the pipeline was blocked completely when the volume concentration of hydrates was up to $68 \%$. The macroscopic structure was still slushlike hydrates before the pipeline was blocked.
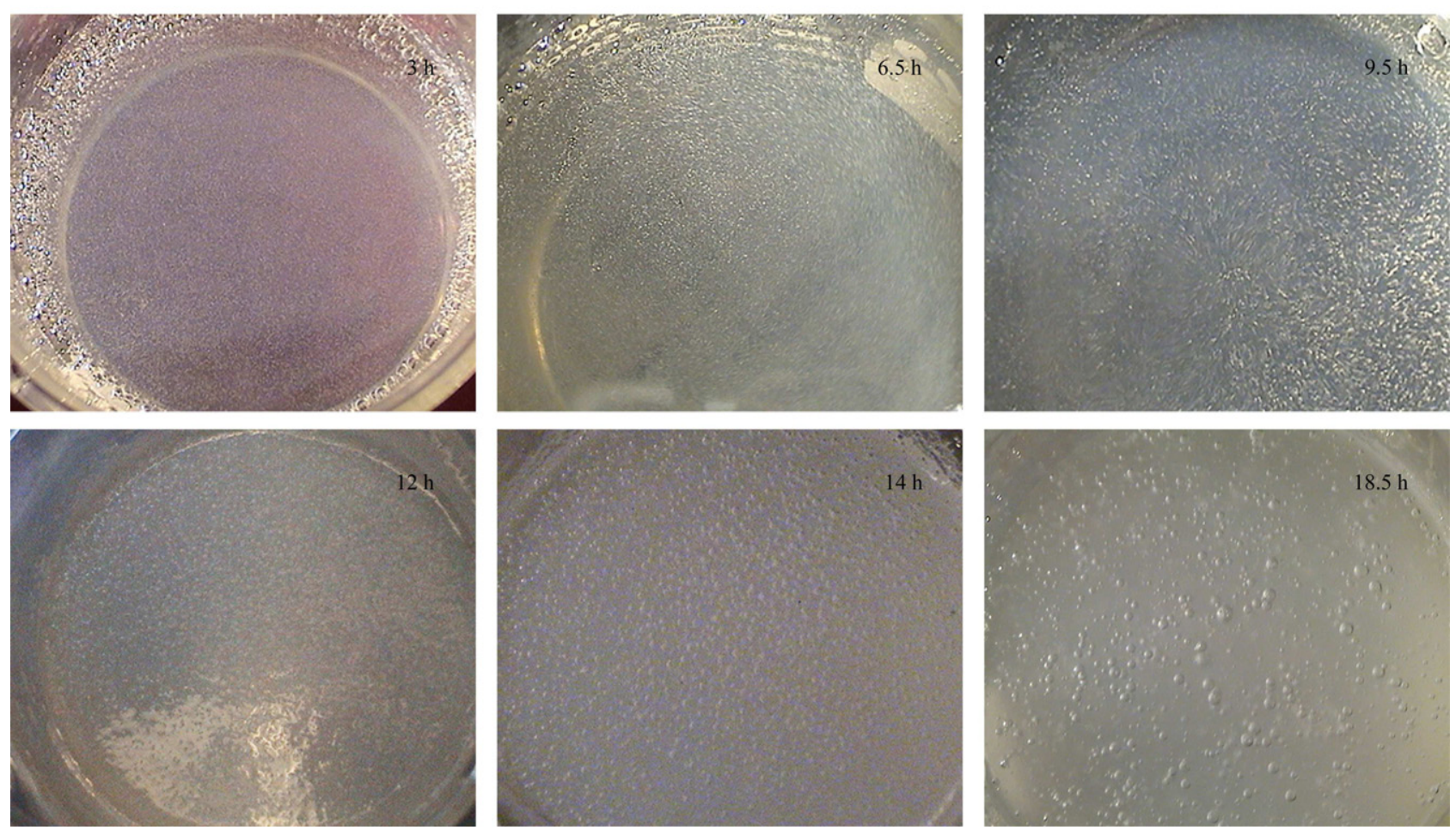

Figure 2. Macroscopic structures of hydrate samples from pipeline as a function of logged time in the forming process of THF hydrate slurry. The water cut in the THF-water solution is $87 \mathrm{wt} \%$ at the beginning of the experiment

From the pictures shown in Figure 2, the appearance of hydrate particles and the transition from slurrylike hydrate to slushlike hydrates are observed visibly. However, the powderlike hydrates were not observed in these experiments because water in these experiments was superfluous compared to THF to form hydrates.

An interesting phenomenon, that hydrates concreted on the cold surface, was observed in the experiments. At the same time the aggregating and depositing of hydrate particles on the wall of the pipeline at low mean velocity were observed in these experiments.

\subsection{Relationship of pressure drop and mean velocity}

Flow properties of different volume concentration of hydrate slurry were studied. The largest $\Phi_{\mathrm{h}}$ in the experiments was $68 \%$, in which the pipeline was completely blocked and the flow experiments could not be performed.

Figure 3 shows the pressure drops as a function of mean velocity of the hydrate slurry in pipeline. As it is seen, the pressure drops increase with the increasing of mean velocities. For each volume concentration of hydrate slurry the pressure drops are larger than those without hydrates in pipeline and the differences increase with the mean velocity especially for those with high solid volume concentration. The plots in Figure 4 show the pressure drops as a function of hydrate volume concentration at steady flow velocities. The pressure drop is almost independent of the hydrate volume concentration when the $\Phi_{\mathrm{h}}$ is less than $50.6 \%$; while there is a remarkable increase of pressure drop with the increase of the hydrate volume concentration when the $\Phi_{\mathrm{h}}$ is larger than $50.6 \%$.

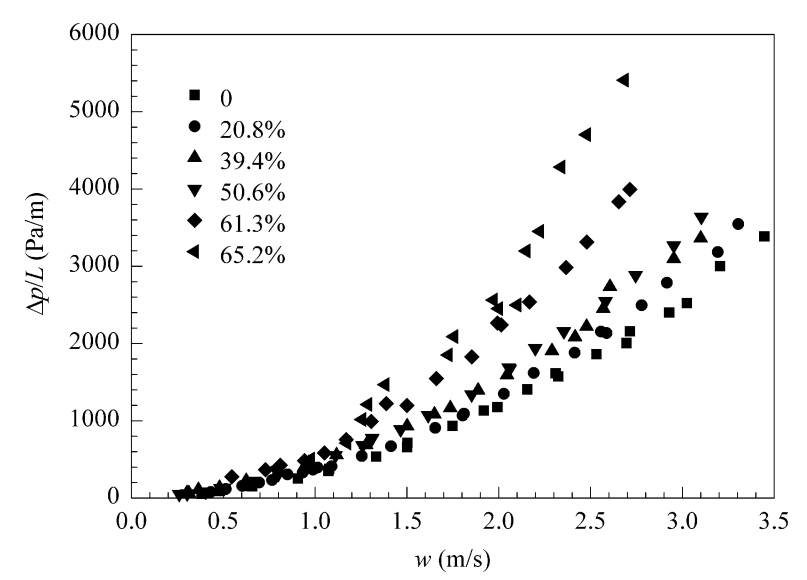

Figure 3. Pressure drops as a function of mean velocity of THF hydrate slurry with different volume concentrations in flow loop

As mentioned above, for the THF slurry system, there is a critical solid volume concentration of $50.6 \%$, at which pressure drops begin to increase substantially; while pressure 
drops are almost independent of the solid volume concentration when it is less than the critical values especially for turbulent flow.

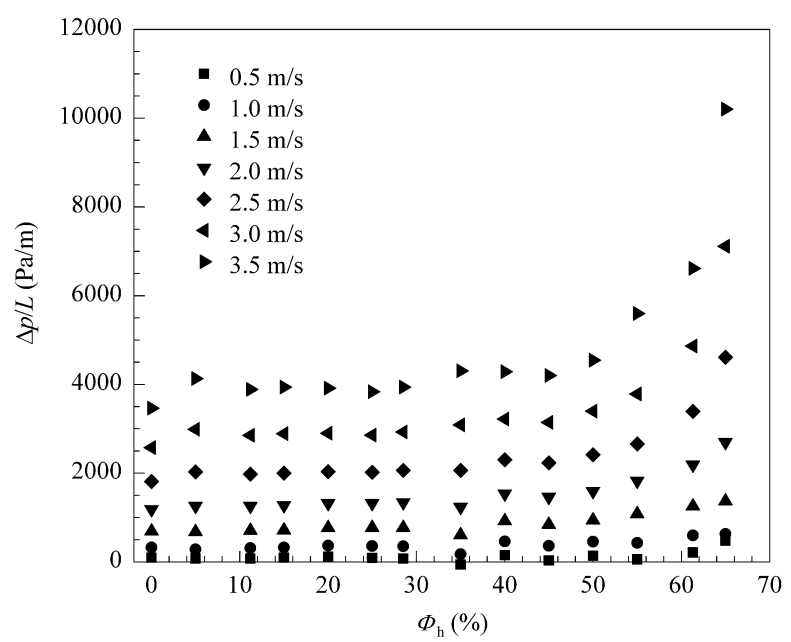

Figure 4. Pressure drops as a function of volume concentration of THF hydrate slurry with different mean velocities in flow loop

The relationship of pressure drop versus solid volume concentration also has been reported by other researchers. In the experiments of oil-based and water-based hydrate slurries [8], it is seen that even for the high concentration (33\%), the frictional pressure drop of the slurry is equal to that of the pure carrying fluids, provided the turbulent flow. This means that no additional pressure drop exists when hydrate slurries are transported in pipelines, compared with pipe transport of pure oil or pure water. However, the critical solid volume concentration was not found in these experiments because of limitation of their mass flow meter used in the experiments for only larger hydrate concentration. While in the flow experiments of THF hydrate/Conroe oil slurries the pressure began to increase substantially at $\sim 10 \%$ when the motor was at $240 \mathrm{r} / \mathrm{min}$ and the pressure drop began to rise at $\sim 18 \%$ hydrates at $520 \mathrm{r} / \mathrm{min}$. Moreover, the trend of increasing flow loop pressure drop with hydrate volume concentration was qualitatively similar to the viscosity increase observed in the laboratory rheological studies [14].

The differences between the critical volume concentrations in the experiments [13] and those in this paper should be caused by the characteristics of the carrying fluids. The Conroe oil has a greatly higher viscosity than water used in this paper, which can intensify the aggregation of the hydrate particles in lower volume concentration. Moreover the low viscosity of water reduces the cutting effects of the flow on the agglomeration of the particles, so the critical volume concentrations of THF hydrate slurries are almost independent of its velocities in pipeline. All these analyzed above suggest that the agglomeration may be the cause of the sudden and steady increase at these critical volume fractions of hydrate. Hydrate particles begin to agglomerate and slushlike hydrates are formed when the solid volume concentration reaches the critical volume concentration.

\subsection{Fanning friction factor of the hydrate slurry}

The Fanning friction factor $(\lambda)$ is a very important parameter in studying the flow characteristics in pipeline, which reflects the flow resistance of the fluid and is defined as following:

$$
\lambda=\frac{D}{2 \rho_{\mathrm{h}} w^{2}} \frac{\Delta p}{L}
$$

Where, $\rho_{\mathrm{h}}$ is the density of THF hydrate and $D$ is the inner diameter of pipeline. Just as most other papers, the Fanning friction factor was calculated through measuring the pressure $\operatorname{drop}(\triangle p / L)$ as well as the mean velocity $(w)$.

The dependence of $\lambda=f(w)$ is shown in Figure 5. As it is seen, the relationship of Fanning friction factor and mean velocity depends on the hydrate volume concentration in slurry. The turbulent flow occurs when there are no hydrates in pipeline with the lowest Reynolds number of 22982, in which Fanning friction factor has very little relation with velocity and is mostly depended on the surface roughness of pipeline. The curve of $\Phi_{\mathrm{h}}=0$ in Figure 5 gives a good example. When some hydrates form in pipeline, the viscosity of the slurry increases and the state of flow at low mean velocity is in the transition zone between laminar flow and turbulent flow, and in this case the Fanning friction factor increases slowly with the increasing mean velocity. And the laminar flow occurs when the volume concentration is higher than $28.5 \%$ at low mean velocity, in which $\lambda$ decreases with increasing mean velocity. Fully turbulent flow occurs and $\lambda$ tend to be constants with all hydrate contents when the velocity reaches $1.5 \mathrm{~m} / \mathrm{s}$. This depends on the volume concentrations in the slurry and is shown in Figure 6.

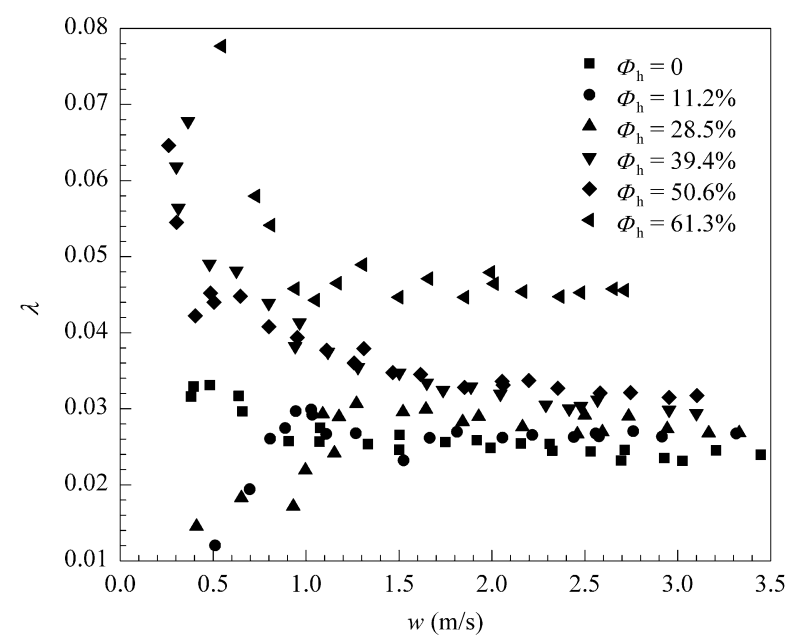

Figure 5. Fanning friction factor of THF hydrate slurry as a function of mean velocity with different solid volume concentration

In order to have a good understanding of Fanning friction factor, the relations to calculate the constant $\lambda$ at large mean velocity were regressed as follows:

$$
\lambda=0.027+5 \mathrm{e}^{\left(\Phi_{\mathrm{h}}-0.074\right) / 0.089} \times 10^{-5}
$$


The variance of regression function in (3) is 0.993 and gives a good fit for the $\lambda$ in the experiments and can be used to estimate the flow pressure drops of the hydrate slurries at large mean velocity with different hydrate volume concentration.

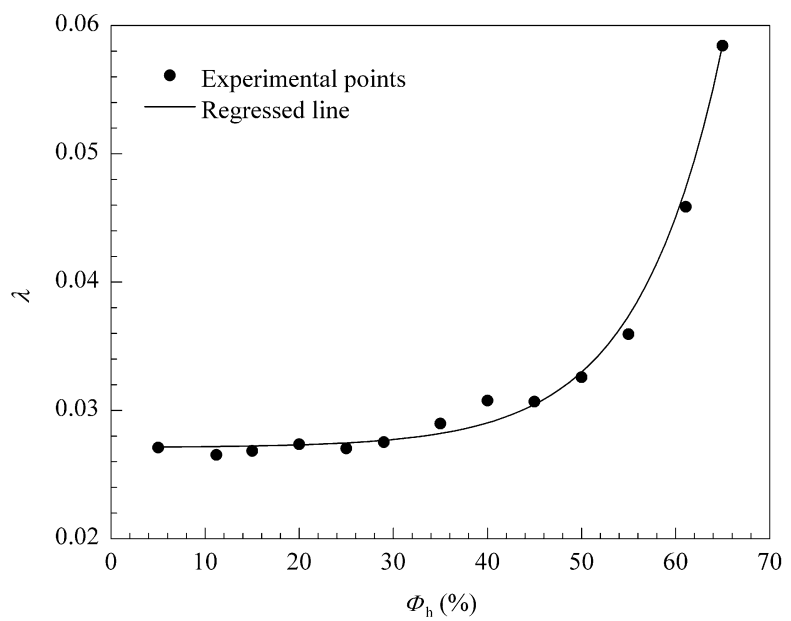

Figure 6. The constant value of Fanning friction factor of THF hydrate slurry as a function volume concentration at high velocities

\subsection{Discussion}

The morphology and the flow behaviors are the key factors to decide whether the pipeline can be run safely or not. According to the experiments, the process of the hydrate blocking the pipeline can be imagined from the analysis of the change of macroscopic structure in pipeline: At first, some hydrate particles are formed in pipeline; slurrylike hydrates condense with ever-increasing hydrates; and then slushlike hydrates, whose large resistance slows the flow velocity of the hydrates slurry, are formed when the solid volume concentration is larger than the critical volume concentration. The temperature of hydrate close to the surface of the pipeline decreases, these hydrates concrete, and this process spreads from the surface to the center of the pipeline and finally the whole pipeline is blocked completely.

On the other hand, the critical volume concentration is decided by the characteristics as well as the velocity of the fluids, which can be estimated by studying viscosity of the slurry according to Turner [14]; While volume concentration in pipeline can be measured through sampling as well as analyzing the components of the fluids.

Now a safe zone, in which the pipeline can be run safely without the hydrate blockage, can be defined by the critical volume concentration for THF hydrate slurry. Pipeline will be free of hydrate blockage when the hydrate volume concentration is lower than the critical volume concentration; while otherwise, pipeline will be easy to be blocked. According to experiments on oil as well as other materials, the critical volume concentration for hydrate slurries also exist and have different values depending on the materials, which may provide some introduction to the further studying on natural gas hydrate slurries to judge whether the pipeline can be run safely or not.

\section{Conclusions}

Systemic researches of morphology and flow behavior of hydrate slurries of THF with different solid volume contents were conducted and some conclusions are proposed as follows:

1) Turbulent flow in pipeline can accelerate the formation of hydrates. Small hydrate particles show at first in pipeline and hydrate slurry is formed with ever-increasing hydrates in pipeline. And then slushlike hydrates are formed in pipeline when the solid volume concentration is larger than the critical one, which leads to the pipeline in a dangerous situation and the pipeline would be completely blocked soon.

2) There is a critical hydrate volume concentration of $50.6 \%$ for THF slurries. Pipeline will be free of hydrate blockage while the hydrate volume concentration is lower than the critical volume concentration; otherwise, pipeline will be easy to be blocked.

3) The effects of mean velocity and the hydrate volume concentration on Fanning friction factor were analyzed. Fully turbulent flow occurs and $\lambda$ tend to be constants with all hydrate contents when the velocity reaches $1.5 \mathrm{~m} / \mathrm{s}$. This depends on the volume concentrations in the slurry and may be regressed to estimate the pressure drops of THF hydrate slurry at large mean velocity.

4) A safe region, which is defined according the critical hydrate volume concentration, was firstly proposed for THF hydrate slurry. The pipeline will be safe when the hydrate concentration is lower than the critical one. And this may give some introduction to the further studying on natural gas hydrate slurries to judge if the pipeline can be run safely or not.

\section{References}

[1] Sloan E D. Fluid Phase Equilib, 2005, 228-229: 67

[2] Yousif M H, Dunayevsky V A. SPE, 30641

[3] Sinquin A. Oil Gas J, 2004, 59(1): 41

[4] Kelland M A, Svartas T M, Andersen L D. J Petrol Sci Eng, 2009, 64(1-4): 1

[5] Kelland M A, Svartaas T M, Øvsthus J, Tomita T, Mizuta K. Chem Eng Sci, 2006, 61(13): 4290

[6] Turner D J, Derek M K, Miller K T. In: The Fifth International Conference on Gas Hydrates, 2005.1116

[7] Fidel-Dufour A, Gruy F, Herri J M. Chem Eng Sci, 2006, 61(2): 505

[8] Derek M K. [MS Dissertation]. Golden: Colorado School of Mines, 2005

[9] Haghighi H. SPE, 107335

[10] Wilson P W. In: The Fifth International Conference on Gas Hydrates, 2005. 568

[11] Wang W C, Fan S S, Liang D Q, Yang X Y. International Journey of Refrigeration, 2008, 31(3): 371

[12] Marinhas S, Delahaye A, Fournaison L. International Journal of Refrigeration, 2007, 30(5): 758

[13] Devarakonda S, Groysman A, Myerson A S. J Cryst Growth, 1999, 204(4): 525

[14] Turner D J, Larry T. In: The Sixth International Conference on Gas Hydrates, 2008 